\title{
Debate about the single etiognostic study
}

\author{
Jan Van den Broeck • Meera Chhagan • \\ Shuaib Kauchali
}

Received: 23 November 2010/ Accepted: 10 February 2011

(C) The Author(s) 2011. This article is published with open access at Springerlink.com

Dr. Miettinen has called for a debate on his ideas about the single etiologic study and the use of trial data for the construction of smooth-in-time risk prediction functions [1]. He did so admittedly as an attempt to influence the possible achievement of a paradigm shift.

Dr. Miettinen is one of the founding fathers of modern epidemiology. Many of the concepts and terms he has introduced have found their way into current epidemiological thinking and discourse, respectively. Why has the same not yet happened with his ideas on the single etiologic study and with the significance of this new paradigm for intervention-prognostic research? Dr. Miettinen wonders and so do presumably many of those who have made a serious effort to study his writings. Although the roots of the single etiologic study can be traced back to Miettinen's 1985 book [2], the formal concept is a bit more recent than the other concepts that are already accepted in epidemiology. So is it simply a matter of time? It might well be, but Dr. Miettinen's concern is to change the'prognostic profile' by opening a debate, which is fair enough and offers an exciting prospect. Remarkably, Pearl, a founder of modern causal theories with wide potential for implementation notably theories and methods of structural causal modelling, recently noted a similar difficulty in getting the

\author{
J. Van den Broeck $(\bowtie)$ \\ Center for International Health, University of Bergen, \\ PO Box 7804, 5020 Bergen, Norway \\ e-mail: jvdbroeck1959@gmail.com \\ M. Chhagan - S. Kauchali \\ Maternal and Child Health Division, Department of Paediatrics, \\ University of KwaZulu-Natal, Durban, South Africa \\ e-mail: chhagan@ukzn.ac.za \\ S. Kauchali \\ e-mail: kauchalis@ukzn.ac.za
}

message down to the masses. His attempt to solution was to produce an article aimed at making the recent advances more accessible through a 'gentle introduction' to the generally unfamiliar complexities of methods such as path analysis [3]. What we are seeing in both cases is a need for highly complex theory to marry with didactics and dissemination strategy. This could mean incorporation into a pedagogical framework that enables future students of epidemiology to embrace this shift for inclusion into mainstream applications.

Epidemiology Journals currently form an important part of the pedagogical framework for epidemiology students. Therefore, in the hope of contributing something to the inception of the debate we would like to ask Dr. Miettinen some questions here about the single etiologic study and its relevance to trial analysis. One of the passages that struck us in the recent paper is where Dr. Miettinen insists that the single etiologic study should not be presented as a unification of the traditional cohort and case-control studies, but that it is a design that differs from both and that should replace any old one. This made us wonder: can or should old etiologic study data be re-analysed in the spirit of the single etiognostic study? If so, would the results in terms of rate ratios be any different, or, when are they expected to be different and why? In the event, would it be possible to provide a practical step-by-step example of how to reanalyse data from a traditional observational follow-up study? If it turns out that re-analysis would be a good idea in more than a minimal proportion of old studies, this could have an enormous impact on epidemiological practice in the coming decennia.

Our own tentative answer is that, yes, re-analysis can often be envisaged because documented population time (in terms of exposure histories) of both study base and of cases arising from it will often be available, whether the 
old etiologic study was of the traditional 'case-control', 'nested case-control' or 'cohort' type. We have no full insight into the procedures needed but we think that there may be two main keys. The first of those is the different handling of etiologic time, which, in the single etiologic study of Miettinen, is negative as from the time of manifestation of case status i.e. as from time of first manifestation of case-defining status in cases, and, end of individual follow-up time in non-cases. This different handling is needed also if the old study was based on a cohort with a planned fixed individual follow-up period. The reversal of etiologic time during re-analysis of traditional cohort data then leads to a situation where the exposure data become left-censored instead of right-censored. The second main key to re-analysis may be the (re)sampling from the study base for the formation of a reference series, which should never be restricted to the noncase domain, and again this would need to hold for reanalysis of all 'old types of design'.

Another question we have relates to the desideratum, mentioned in the recent paper [1], for the case series to be much smaller than the reference series in the suggested additional analysis of trial data. What would be a good operational definition of 'much smaller' in these matters? We think that, especially in smaller trials, the outcome is often a relatively frequent event (in terms of incidence) so that the base will not be that much bigger than the case series? Would there be any recommended approach to constructing a prognostic function in such instances? On the matter of advancing the quality of intervention studies we agree that the paradigm of the single etiognostic study would not only advance quality but also cost efficient use of the research infrastructure invested in intervention studies. Questions here revolve around how best to abstract the base series, bearing in mind both left- and right censoring of the 'source population' in intervention studies.

As always, the ideas of Dr. Miettinen are well worth studying and debating. We are thrilled by the prospect of an interesting debate but, for many, (examples of) implementation will be a condition for deeper understanding.

\section{References}

1. Miettinen OS. Etiologic study vis-à-vis intervention study. Eur J Epidemiol. 2010; 25:671-5.

2. Miettinen OS. Theoretical Epidemiology. New York: Delmar Publishers; 1985, ISBN 0-8273-4313-2, pp. 1-359.

3. Pearl J. An introduction to causal inference. Int $\mathbf{J}$ Biostat. 2010; 6(7):1-59.

\section{Professor Miettinen Replies:}

To propose, as I did [1], a paradigm shift in our thinking about ideal etiologic and intervention studies-in what we take to be the essence of each of these-is to say something that inherently invites a response from any critical teacher of epidemiological research. The implicit questions to any such teacher were: Will you start teaching the etiologic study as the necessary replacement for 'cohort' and 'casecontrol' studies? and: will you teach the use of this study structure on the (usual type of) data from intervention trials - with the proposed, novel variant of logistic regression replacing Cox regression and leading to prognostic probability functions? Before answering these questions, however, such a teacher may well see the need to have me answer some questions of theirs.

I was, thus, astonished and disappointed to find that the initial response to my explicit (sic) call for public discourse on these matters in this journal was a single Letter to the Editor.

In that Letter, Van den Broeck et alii [2] seem to implicitly express agreement with what I said about etiologic and intervention studies. But they describe my propositions as constituting a 'highly complex theory,' presenting notable challenges for didactics and other dissemination; and they thus ask two didactics-related questions about it: '[C]an or should old etiologic study data be re-analysed in the spirit of the single etiognostic study?' and 'What would be a good operational definition of [suitably large for the base series relative to the size of the case series in the proposed type of logistic regression on data from intervention trials]?'

To these colleagues I say, for orientation, that I regard 'highly complex theory' as much too grandiose a characterization of the novelties I advocate.

'Re-analyses' of data from already-published 'cohort' studies would be feasible and instructive; and they would be particularly valuable in the context of studies in which, as is usual, the source-cohort's follow-up is of long (and hence variable) duration. To be borne in mind in these is that the population-time of the cohort's follow-up constitutes only the source base, and that therefore the first-stage case and base series from it need to be reduced to those from the actual study base (each person-moment in it actually representing the domain of the object of study and presence of one of the contrasted histories). The translation of 'cohort' studies to genuine etiologic studies is fully instructive of the general essence (singular) of etiologic studies, including in the context of dynamic source populations: commitment to a source population, specifically to the source base formed by its course over a particular span of time; etc. 
In a 're-analysis' (re-synthesis) of the data from an etiologic study with a cohort-type source population or from an intervention trial, the contextual point of note is that there is no material cost (marginal) to however large an expansion of the base series. Hence, it should be made large-enough to abstract practically all of the (comparative) information in the study base. A 100 -fold sample already is suitably large, a 1,000-fold sample better to an immaterial extent.

Concerning their Letter, Van Den Broeck et alii express 'the hope of contributing something to the inception of the [public discourse]'; and they do just that, uniquely. I thank them for this and very much hope others will follow their example, whether spontaneously or by the Editor's solicitation.
Open Access This article is distributed under the terms of the Creative Commons Attribution Noncommercial License which permits any noncommercial use, distribution, and reproduction in any medium, provided the original author(s) and source are credited.

\section{References}

1. Miettinen OS. Etiologic study vis-à-vis intervention study. Eur J Epidemiol. 2010;25:671-5.

2. Van de Broeck J, Chhagan M, Kauchali S. Debate about the single etiognostic study. Letter to the Editor. Eur $\mathrm{J}$ Epidemiol. 2011;26(4):339-41. 